Abb. 1 Statue des Hippokrates: Die Schriftsammlung „Corpus Hippocraticum" beschreibt die theoretische Grundlage der Humoralmedizin. Doch nur wenige Werke aus der Sammlung lassen sich wirklich auf Hippokrates selbst zurückführen. Foto: ๑ Adobe Stock/markara

\title{
Humoralpathologie: Grundlage für Prävention und Therapie
}

\section{ÜBERLIEFERTE WISSENSSCHÄTZE aus Antike, Mittelalter und Moderne: Gegensatz- und Ähnlichkeits-} prinzip, Temperamenten- und Säftelehre, traditionelle und rationale Phytotherapie

Iris Eisenmann-Tappe

DIE KLOSTERMEDIZIN fiel in eine Epoche, in der man auf Traditionen baute und eine hohe Achtung vor Autoritäten hatte. Als solche galten nicht nur kirchliche Würdenträger, sondern auch charismatische und berühmte antike Ärzte wie Hippokrates (ca. 460-370 v. Chr.) und Galen (ca. 129-205 n. Chr.). Denn das gesamte medizinische Wissen des Mittelalters wurzelt in der Antike. Dem Fleiß unzähliger Mönche beim Kopieren, Bewahren und Verwalten der damals schon alten, hoch geschätzten Wissensliteratur verdanken wir unsere Einsicht in die Ursprünge der heutigen Medizin.

\section{Zweitausend Jahre Temperamenten-} und Säftelehre

Man ging in der Medizin des Mittelalters davon aus, dass jeder Mensch von Geburt an ein bestimmtes Temperament ausprägt und damit überwiegend als Choleriker, Melancholiker, Phlegmatiker oder Sanguiniker einzustufen ist. Hervorgerufen werden diese Unterschiede durch das Vorherrschen eines der vier Körpersäfte (Humores) - Chole (Gelbe Galle), Melanchole (Schwarze Galle), Phlegma (Schleim) oder Sanguis (Blut). Diese theoretische Grundlage der Humoralmedizin wurde im „Corpus 
Hippocraticum “ beschrieben, einer Sammlung von medizinischen Schriften aus der Zeit zwischen dem 6. Jh. vor und dem 2. Jh. nach Christus. Nur wenige Werke stammen jedoch von Hippokrates selbst.

Durch das ganze Mittelalter hindurch war es für die Menschen von großer Bedeutung, ihr eigenes Temperament zu kennen. Ziel für jedermann war, seine Lebensweise danach auszurichten und dadurch ein Ungleichgewicht in der persönlichen Säftemischung zu vermeiden. Denn eine solche Disharmonie (Dyskrasie) wurde als Hauptursache von Krankheiten angesehen.

\section{Erkenne dich selbst und deine}

\section{Bedürfnisse, vermeide Extreme und Übertreibungen in jeder Richtung.}

\section{Sechs nicht natürliche Dinge als Meilensteine gesunden Lebens}

Damit die Menschen die als gültig betrachteten Theorien auch umsetzen konnten, verfasste man im Mittelalter sogenannte Regimen sanitatis (zu Deutsch: Gesundheitsregeln) mit kompakt formulierten Empfehlungen. Wegbereiter für diese ersten Gesundheitsratgeber war die „Epistula de observatione ciborum“ des byzantinischen Arztes Anthimus aus dem 6. Jh. nach Christus, die im „Lorscher Arzneibuch“ aus dem 8. Jahrhundert überliefert ist. In den Regimen sanitatis wurden die sogenannten Sex res non naturales (sechs nicht natürliche Dinge) erläutert. Diese galten als entscheidend für die Bewahrung der Gesundheit, da sie nicht als "natural“ (sozusagen gottgegeben und damit unabwendbar), sondern als vom Menschen verantwortlich steuerbar betrachtet wurden:

1. Aer - gesunde Luft

2. Motus et quies - Bewegung und Ruhe

3. Cibus et potus - Essen und Trinken

4. Somnus et vigilia - Schlafen und Wachen

5. Repletio et evacuatio - Füllung und Ausleitung

6. Accidentia animae - Emotionen beziehungsweise

psychische und seelische Verfassung

Jeder ganzheitlich denkende Therapeut wird heute die Bedeutung dieser sechs Faktoren bestätigen. Interessant für die heutige Zeit ist dabei vor allem der Aspekt der individuellen Steuerbarkeit: Wir haben nämlich im Hinblick auf alle diese Punkte an Selbstbestimmungsmöglichkeiten verloren. Man denke beispielsweise beim Faktor Aer an die aktuelle Diskussion über Dieselabgase in den Städten oder bei Cibus et potus an die Kontaminierung des Grundwassers und der Nahrungsmittel mit Umweltchemikalien wie Nitrat oder Pestiziden.

Repletio et evacuatio spiegeln das Streben nach einer geregelten Verdauungs- und Stoffwechseltätigkeit wider. Füllung

\section{KURZ GEFASST}

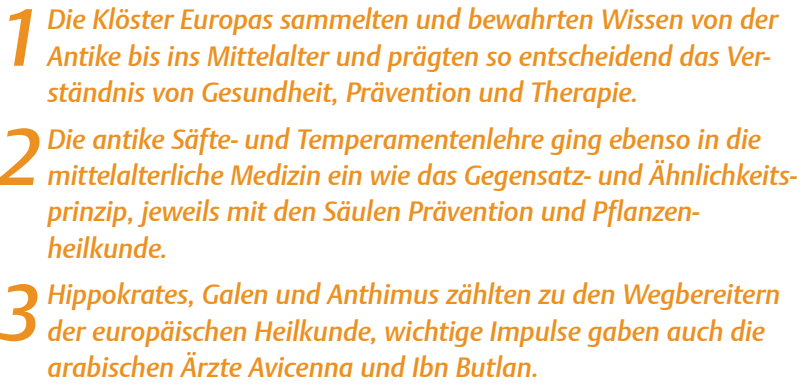

und Entleerung des Verdauungstrakts sollen in einem gesunden Rhythmus des Stoffwechsels erfolgen, der auch durch Arzneimittel oder Bäder beeinflusst werden kann. Wie wichtig diese Faktoren für Gesundheit und Wohlbefinden sind, führt uns aktuell die hohe Prävalenz des Reizdarmsyndroms (siehe S. 2227) in Deutschland vor Augen: Laut der Deutschen Reizdarmselbsthilfe e.V. ist etwa jeder Sechste von chronischen funktionellen Verdauungsstörungen betroffen.

Unregelmäßige Arbeitszeiten oder Stress sind heute für viele Menschen nicht zu umgehen, Bewegungsmangel ist oft mit chronischem Zeitmangel verbunden. Dass wir solch wichtigen Gesundheitsfaktoren wie Schlaf- und Wachrhythmus und der Pflege der seelischen Gesundheit nicht die notwendige Beachtung zukommen lassen, zeigt Folgen. So sind beispielsweise in Deutschland laut dem Depressionsatlas der Techniker Krankenkasse seit dem Jahr 2000 die Arbeitsausfälle wegen behandlungsbedürftiger Depressionen um fast $70 \%$ angestiegen. Demnach sind seelische Erkrankungen heute der zweithäufigste Grund für Krankmeldungen.

Prävention hatte in der mittelalterlichen Medizin einen hohen Stellenwert, weil die Auswahl therapeutischer Mittel wesentlich geringer war als heute. Ärzte wie Patienten im Mittelalter bemühten sich deshalb um eine innere Harmonie der Säfte, die Eukrasie. Sinngemäß lautet der in diesem Wort zusammengefasste Rat zur Erhaltung der Gesundheit: Erkenne dich selbst und deine Bedürfnisse, vermeide Extreme und Übertreibungen in jeder Richtung.

\section{Avicenna als Wegbereiter der humoralmedizinischen Diagnostik}

Abu Ali al Hussein Ibn Abdillah Ibn Sina (ca. 980-1037), auch Avicenna genannt und in jüngerer Zeit durch die Verfilmung des Bestsellers „Der Medicus“ wieder bekannt geworden, erstellte um das Jahr 1030 sein medizinisches Hauptwerk, den „Canon medicinae“. Der brillante persische Arzt, Denker und Universalgelehrte systematisierte das Wissen seiner Zeit. Der „Canon medicinae" wurde bereits im 12. Jahrhundert n. Chr. ins Lateinische übersetzt und nahm über Jahrhunderte als Standardwerk großen Einfluss auf die europäische Medizin. Avicenna hatte, aufbauend auf Galens Humoralpathologie, ein ausgefeiltes Ana- 
mnesesystem entwickelt. Patienten wurden nicht nur nach ihrem körperlichen und seelischen Wohlbefinden befragt. Auch der Einfluss von Alter, Klima und Umwelt wurde mit einbezogen.

\section{Aspekte der humoralmedizinischen Diagnostik}

Mit der Fähigkeit zur Typisierung nach den humoralmedizinischen Temperamenten sowie der Erstellung eines individuellen Gesundheitsprofils steht und fällt die Anwendung der Humoralpathologie bis heute in der Praxis. Die Heilmittel wurden dabei individuell auf jeden einzelnen Patienten abgestimmt. Beurteilt wurden:

" äußerer Eindruck, insbesondere Statur, Gesicht, Augen, Haut, Haare, Nägel

- Verhalten, vor allem Bewegung, Stimme, Sprache, Atmung, emotionale Verfassung

- Ergebnisse der körperlichen Untersuchung (Palpation, Pulsmessung, Harnuntersuchung und Beurteilung der Zunge)

Ein TCM-Therapeut wird unmittelbar die Parallelen zum Vorgehen in der Traditionellen Chinesischen Medizin bemerken. Avicenna war auch innovativ bei Heilverfahren. So verordnete er Musiktherapien oder forderte Patienten zu Reisen oder Spaziergängen am Meer auf, um sie aus alten Denk- und Verhaltensmustern zu befreien. Eine integrierte Bewegungstherapie wie das Qi Gong in der TCM entwickelte die Humoralmedizin allerdings nicht. Ein Grund dafür mag sein, dass man pathologische Stagnationen weniger durch Ganzkörperbewegung, sondern vor allem durch Motilitätsförderung im Inneren des Körpers lösen wollte. Dies geschah durch teils drastisch anmutende Ausleitungsverfahren wie den Aderlass oder die Erregung von Durchfall, Erbrechen oder auch Niesen.

Das fünfte Buch im „Canon medicinae“ enthält die Beschreibung von über 600 Heilmitteln. Der Großteil der Medikamente ist pflanzlichen Ursprungs und demonstriert die weit entwickelte Phytotherapie dieser Zeit. Allein dieses Werk bietet noch viele Anregungen für die moderne pharmakologische Forschung: Wir haben bei weitem noch nicht alle Wissensschätze gehoben. Wie aber wählt der Therapeut nach Avicennas Lehre die passenden Arzneimittel für seinen Patienten aus?

\section{Similia- und Contraria-Prinzip: mittelalterliche Vorläufer der Homöopathie}

Zwei antagonistische Heilungsansätze waren zu Avicennas Zeiten im humoralpathologischen System gebräuchlich: Similia similibus oder Contraria contrariis curantur: Ähnliches möge durch Ähnliches oder Entgegengesetztes durch Entgegengesetztes geheilt werden. Dies betraf Nahrungsmittel wie auch Arzneimittel: In Antike und Mittelalter wurde kein grundsätzlicher Unterschied zwischen beidem gemacht. Im Sinne des SimiliaPrinzips nahm man beispielsweise zur Vermehrung des Blutes rote Speisen zu sich, darunter rotes Fleisch, Rote Bete und rotes
Beerenobst. Manches erscheint auch aus heutiger Sicht plausibel. So unterstützt rotes Fleisch beispielsweise durch seine hohen Gehalte an Eisen und Vitamin $\mathrm{B}_{12}$ die Blutbildung.

In anderen Fällen half das Prinzip nach heutigem Ermessen nicht weiter. So erklärt sich die über mehrere Jahrhunderte ungebrochene Beliebtheit der weißen Speise Blancmanger (Mandelsulz) recht einfach durch das Similia-Prinzip: Die sämige Beschaffenheit dieser Zubereitung aus weißem Reis, zerkleinertem Hühnerfleisch und Mandelmilch sollte aufgrund der Ähnlichkeit in Konsistenz und Farbe eine reichliche Produktion der männlichen Samenflüssigkeit fördern. In der Konsequenz wurde der Blancmanger gesüßt - eine für den heutigen Geschmack eher irritierende Würzung. Denn nach der Theorie der Viersäftelehre befördert Zucker im Körper die Vermehrung von Phlegma (Schleim), also auch der Samenflüssigkeit. Auch wenn uns heute solche Zusammenhänge unsinnig erscheinen, ist dies ein gutes Beispiel für die zur damaligen Zeit selbstverständliche Auswahl von Nahrungsmitteln zu gesundheitlichen Zwecken. Das Similia-Prinzip der Humoralpathologie und volkstümlichmagisches Denken sind Wurzeln der Signaturenlehre, die in Antike und Mittelalter schon weite Anwendung fand, bevor Paracelsus sie schriftlich formulierte.

Wer heute Homöopathie anwendet, kennt das Similia-Prinzip. Samuel Hahnemanns Heilmethode, die er ab 1796 veröffentlichte, wurzelt so betrachtet in der humoralpathologischen Theorie und war im Grunde bereits im „Corpus Hippocraticum“ dargestellt worden, allerdings ohne die Idee einer therapiewirksamen Verdünnung der Wirkstoffe. Die Signaturenlehre lehnte Hahnemann allerdings ab, da sie sich vor allem auf die Ähnlichkeit von Zeichen, nicht aber von Krankheitssymptomen bezog.

\section{Von Avicenna bis Ibn Butlan: Einflüsse der Orientalischen Medizin}

Auch die gegenteilige Auffassung, das Contraria-Prinzip, findet sich bereits im „Corpus Hippocraticum“. Um beispielsweise Beschwerden durch einen kalten (dyspeptischen, zu trägen, schlecht verdauenden) Magen zu mildern, wurde der reichliche Verzehr von heiß wirkendem Knoblauch empfohlen. Um das Contraria-Prinzip anzuwenden, musste man also wissen, welche Komplexion die Nahrungs- und Arzneimittel jeweils aufwiesen.

Unter Komplexion ist die Charakterisierung des Mittels als warm oder kalt, andererseits als trocken oder feucht zu verstehen. Zur Einstufung der Stärke der jeweiligen Eigenschaft benutzte man eine Viererskala mit dem 1. als dem schwächsten, sowie dem 4. als dem stärksten Grad. Der Knoblauch beispielsweise galt als warm im 4 . und trocken im 3. Grad.

Für die Menschen des Mittelalters gehörte dies zum Allgemeinwissen, unterstützt durch die erwähnte Ratgeberliteratur. Ein schönes Beispiel dafür sind die in deutscher Fassung ab 1533 in Druckform erhältlichen „Schachtafelen der Gesuntheyt“ [1] (siehe „Die ersten Tabellen der Medizingeschichte“). 


\section{Die ersten Tabellen der Medizingeschichte}

Bei den „Schachtafelen der Gesuntheyt“ handelt es sich um ein übersichtliches Tabellenwerk, in dem Leser die Wirkungen von Genuss- und Arzneimitteln, aber auch zum Beispiel die Effekte von Bewegung, Schlaf, Kleidung oder Jahreszeiten nachschlagen konnten. Der ursprüngliche Verfasser dieser synoptischen Tabellen war der arabische Arzt Ibn Butlan (gestorben 1065), der das Medizinische Hausbuch „Tacuinum sanitatis“ als Rahmenwerk Mitte des 11. Jahrhunderts verfasste. Sein Ziel war, den gesundheitlichen Wert sämtlicher Bereiche des menschlichen Lebens und der Umwelt darzustellen. Er beschrieb im Vorwort seine Motivation für die Schaffung eines übersichtlichen Tabellenwerks: „Denn die Menschen wollen von den Wissenschaften nichts anderes als wirksame Hilfe, nicht aber Beweise und Definitionen ...Daher ist es unsere Absicht in diesem Buche, umständliches Gerede abzukürzen ..."

Wegen des hervorragenden Rufs der orientalischen Heilkunst war auch dieses Werk bald ins Lateinische übersetzt worden und so dem abendländischen Mittelalter zugänglich.

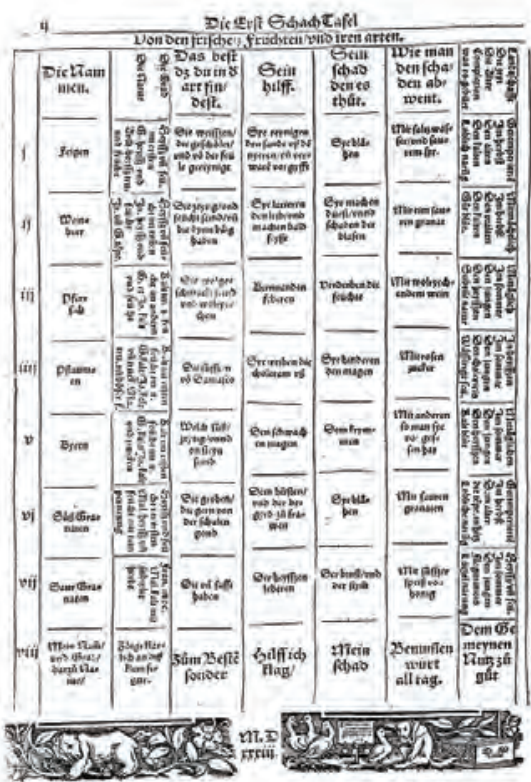

Abb. 2 Die erste Schachtafel aus dem medizinischen Hausbuch „Schachtafelen der Gesuntheyt“ von 1533. 


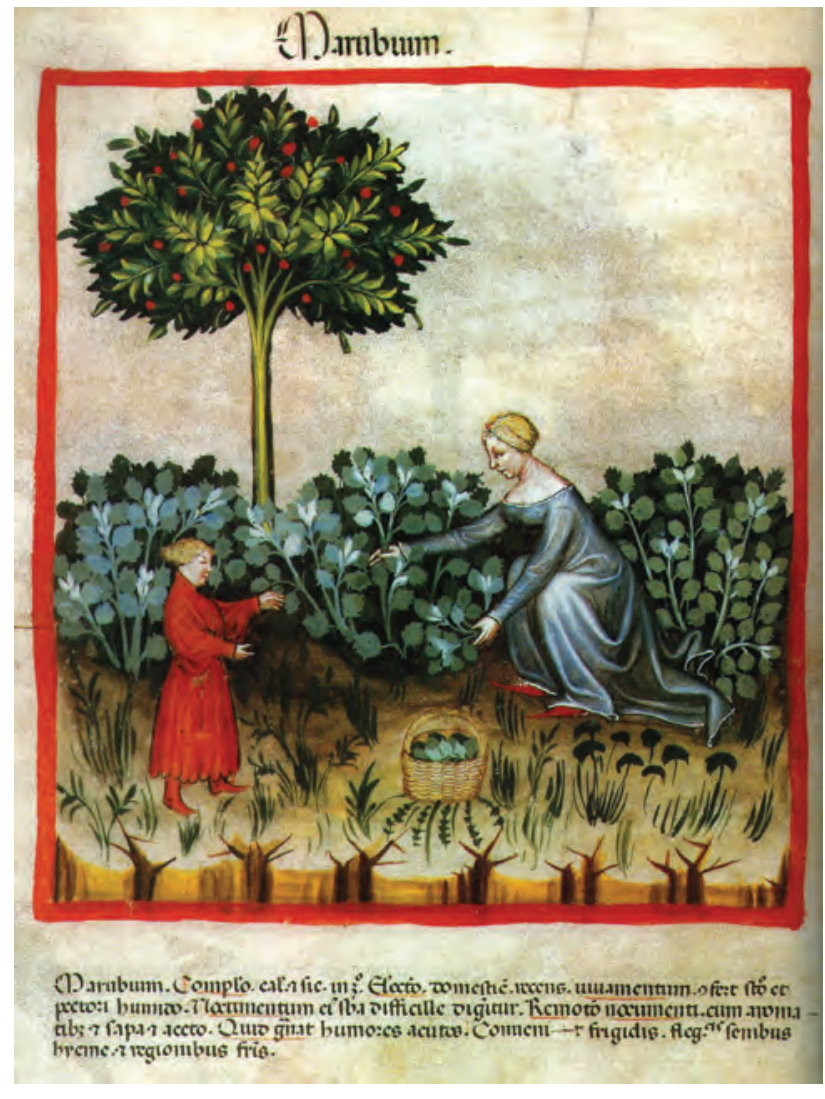

Abb. 3 Darstellung von Andorn (Marrubium) im „Tacuinum sanitatis“. Um seinen Nutzen als medizinisches Hausbuch noch zu vergrößern, war das „Tacuinum sanitatis“ im 14. Jahrhundert von einem unbekannten Meister mit Miniaturen bebildert worden. Das hier gezeigte Beispiel stellt die Blattform und das silbrige Laub des Andorns dar.

\section{"Denn die Menschen wollen von den Wis-} senschaften nichts anderes als wirksame Hilfe. “

\section{aus „Schachtafelen der Gesuntheyt"}

\section{Vergleichende Beurteilung mittelalterlicher Schriften am Beispiel Andorn}

Am Beispiel des Andorns (Marrubium vulgare, siehe auch Heilpflanzenporträt auf S. 56-59) lässt sich der Beitrag mittelalterlicher Schriften zum heutigen Heilpflanzenwissen verdeutlichen. So schreibt Ibn Butlan in den „Schachtafelen der Gesuntheyt“: „Komplexion: warm und trocken im 3. Grad. Vorzuziehen: im Haus gewachsen und frisch. Nutzen: gut für den Magen und für die feuchte Brust. Schaden: Seine Substanz ist schwer verdaulich. Verhütung des Schadens: mit wohlriechenden Stoffen, Mostsaft und Essig. Was er erzeugt: scharfe Säfte. Zuträglich für Menschen mit kalter Komplexion, für Phlegmatiker, Greise, im Winter und in kalten Gegenden.“ [1]
Hildegard von Bingen (1098-1179, siehe S. 60-63) schreibt zwischen 1150 und 1160 ohne Kenntnis von Ibn Butlans Schriften in ihrer Physica: „Der andron ist warm und hat reichlich Saft. (...) wessen Kehle krank ist, der koche andron in Wasser, seihe das gekochte Wasser durch ein Tuch, setze die doppelte Menge Wein hinzu und lasse das Ganze unter Zugabe von reichlich Schmalz nochmals in einer Schüssel aufkochen. Das trinke er oft und er wird hinsichtlich seiner Kehle Heilung finden (...). Wer kranke oder gebrochene Eingeweide hat, koche andron mit Wein unter Hinzufügung von reichlich Honig, lasse es nach dem Kochen in dem Topf und trinke davon oft, nachdem es abgekühlt ist. Es heilt die Eingeweide.“ In beiden, voneinander unabhängigen Texten werden also zwei der heute anerkannten Indikationen für die Anwendung von Andornkraut bereits genannt: als Expektorans bei erkältungsbedingtem Husten und als Mittel gegen dyspeptische Beschwerden (Hildegards Rat, den sehr bitteren Andorntee reichlich zu süßen, mag auch der heutige Therapeut seinen Patienten zur besseren Compliance mit auf den Weg geben).

Durch solche vergleichende Texterschließung, Einflüsse der Volksheilkunde und später gezielte Forschung entstand in der Moderne schließlich die rationale Phytotherapie (siehe Kasten). Diese Entwicklung verlief allerdings nicht kontinuierlich.

\section{Überlieferungen aus der Antike galten bis ins 19. Jahrhundert}

Die Medizin der Antike mit ihren Schwerpunkten Prävention und Pflanzenheilkunde blieb dank der Bewahrung durch die Klostermedizin erhalten und erfreute sich im Abendland bis um 1800 großer Wertschätzung. Mit der zunehmenden Entwicklung synthetischer Arzneistoffe gegen Ende des 19. Jahrhunderts wurde die bis dahin dominierende Pflanzenheilkunde jedoch mehr und mehr von der Behandlung mit rascher und gezielter wirksamen chemisch-synthetischen Medikamenten abgelöst. Die Bedeutung der Prävention geriet aus dem Blickfeld. Was die Pflanzenheilkunde anbetrifft, so bezeichnete noch Anfang der 1990er-Jahre die Deutsche Gesellschaft für Pharmakologie und Toxikologie „die Phytotherapie als eine wissenschaftlich zurückgebliebene Form der Arzneitherapie, welche eher historisch zu verstehen ist...“ [2]

\section{Exkurs: Sind rationale Phytotherapie und Homo̊opathie vereinbar?}

Die moderne rationale Phytotherapie ist keine Alternativmedizin, sondern Teil der naturwissenschaftlich orientierten Schulmedizin. Damit unterscheidet sie sich grundsätzlich von der Homöopathie, die ihre Therapeutika nicht nach naturwissenschaftlichen Prinzipien auswählt. Die prinzipielle Unvereinbarkeit beider Behandlungsverfahren hält heute viele Therapeuten nicht davon ab, sie kombiniert einzusetzen: 60000 Ärzte wen- 
- DEFINITION

\section{Die rationale Phytotherapie}

Als Phytotherapie bezeichnet man die Heilung, Linderung und Vorbeugung von Krankheiten durch Arzneipflanzen, deren Teile (zum Beispiel Blüten oder Wurzeln), Bestandteile (zum Beispiel ätherische Öle) und / oder Zubereitungen (unter anderem Trockenextrakte, Tinkturen, Presssäfte). Arzneimittel der Phytotherapie werden als Phytopharmaka bezeichnet. Für deren Zulassung nach dem Arzneimittelgesetz (AMG) müssen Wirksamkeit und Unbedenklichkeit anhand von produktspezifischen, präklinischen, pharmakologischen und toxikologischen Studien oder anhand von bibliografischen Unterlagen der Sachverständigen-Kommission E erbracht werden. Die strengen Zulassungsanforderungen entsprechen den Vorgaben für chemisch-synthetische Arzneimittel [5]. den in Deutschland laut dem Zentralverein homöopathischer Ärzte e.V. komplementärmedizinische Verfahren an. Zum Vergleich: Derzeit sind 47000 Heilpraktiker in Deutschland tätig [3].

Samuel Hahnemann selbst hat sich gegen derartige Kombinationen verwahrt: „Es giebt nur zwei Haupt-Curarten: diejenige welche all' ihr Thun nur auf genaue Beobachtung der Natur, auf sorgfältige Versuche und reine Erfahrung gründet, die (vor mir nie geflissentlich angewendete) homöopathische, und eine zweite, welche dieses nicht thut, die allöopathische." [5] Weiter schreibt er: „Jede steht der andern gerade entgegen und nur wer beide nicht kennt, kann sich dem Wahne hingeben, dass sie sich je einander nähern könnten oder wohl gar sich vereinigen liessen, kann sich gar so lächerlich machen, nach Gefallen der Kranken, bald homöopathisch, bald allöopathisch in seinen Curen zu verfahren; diess ist verbrecherischer Verrath an der göttlichen Homöopathie zu nennen! “ [4]

Die Anwender im Mittelalter hatten jedoch kein Problem mit der theoretischen Gegensätzlichkeit des Contraria- und SimiliaPrinzips: Sie wandten beide an. Ibn Butlan hat in seinem Tacuinum bereits einen Vorschlag unterbreitet, wie man beide Prinzipien synergistisch vereinbaren könnte: Bei Gesundheit solle man nach dem Similia-Prinzip vorbeugen, bei Krankheit beziehungsweise Dysbalance das Contraria-Prinzip anwenden. Ein gutes Beispiel für Pragmatismus im Sinne des Patienten.

\section{Verwendete Literatur}

[1] Schott H. Schachtafeln der Gesundheit. Deutsche Fassung von Ibn Butlans Taqwim es-sihha. Straßburg: 1533

[2] Deutsche Gesellschaft für Pharmakologie und Toxikologie. Mitteilungen Nr. 12, Sonderheft März 1993: 22-23

[3] Bund Deutscher Heilpraktiker (BDH) e. V. Zehn Fakten zum Heilpraktikerberuf. https://www.heilpraktiker-fakten.de/heilpraktikerfakten/zehn-fakten-zum-heilpraktikerberuf/; Stand: 29.05.2018

[4] Hahnemann S. Organon der Heilkunst. 6. Auflage (Nachdr.). Kandern: Narayana; 2011: §52

[5] Schilcher H. Leitfaden Phytotherapie. München: Elsevier; 2016

Dieser Artikel ist online zu finden:

http://dx.doi.org/10.1055/a-0617-6866

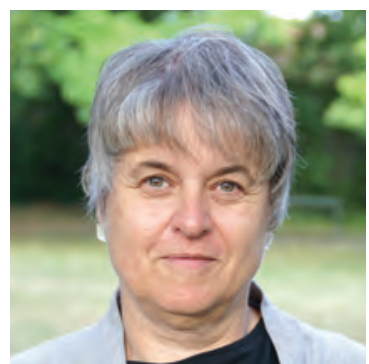

\section{HP Dr. rer. nat. Iris Eisen-}

\section{mann-Tappe}

\section{Gartenstr. 39}

97286 Sommerhausen

E-Mail: info@heilpraktikerin-tappe.de

Iris Eisenmann-Tappe ist Diplom-Biochemikerin. Seit 1996 war sie selbstständig mit einer Praxis zur pädagogischen Therapie von Dyslexie und Lernstörungen. Seit 2013 betreibt sie als Heilpraktikerin eine eigene Praxis mit den Schwerpunkten Ernährungsheilkunde, Pflanzenheilkunde und Darmgesundheit. Seit 2015 ist sie für die Forschergruppe Klostermedizin tätig als Dozentin für Phytotherapie, Stoffwechsel und Ernährung. 